\title{
Effect of Fractal Tones on the Improvement of Tinnitus Handicap Inventory Functional Scores among Chronic Tinnitus Patients: An Open-label Pilot Study
}

\author{
Patricia Simonetti $^{1} \quad$ Laura Garcia Vasconcelos $^{1}$ Jeanne Oiticica ${ }^{1}$ \\ ${ }^{1}$ Department of Otorhinolaryngology, Faculdade de Medicina da \\ Universidade de São Paulo, São Paulo, SP, Brazil \\ Address for correspondence Jeanne Oiticica, MD, PhD, Departmento \\ de Otorrinolaringologia, Universidade de São Paulo, Alameda dos \\ Jurupis 657, CJ 72, Indianópolis, São Paulo, SP, 04088-002, Brazil \\ Int Arch Otorhinolaryngol 2018;22:387-394. \\ (e-mail: jeanneramalho@uol.com.br).
}

\begin{abstract}
Introduction Music-based sound therapies become recently a trend in the treatment of tinnitus. Few publications have studied the therapeutic use of fractal tones to treat chronic tinnitus.

Objective The aim of this study was to determine the benefits of using fractal tones as a unique sound therapy for chronic tinnitus sufferers.

Methods Twelve participants were recruited; however, six could not be assigned. At baseline and at 1, 3 and 6 months, the participants were provided with the Tinnitus Handicap Inventory (THI) and Hearing Handicap Inventory for Adults (HHIA) questionnaires, and their visual analog scale score was recorded. Tinnitus pitch and loudness matching was performed before fitting and after 6 months of use.

Results The visual analog scale (VAS) score, pitch and loudness matching and minimal masking levels did not result in significant improvement at the end of the treatment. The mean THI measured at baseline was 45 , and the final one was 25 . A paired sample $t$-test showed that this 20-point difference was statistically significant.

Conclusions We measured the benefits of using fractal tone therapy for the treatment of chronic tinnitus and found that most of the benefits were in the THI functional domain, which includes concentration, reading, attention, consciousness, sleep, social

Keywords

- tinnitus

- fractal

- music therapy

- THI activities, and household tasks. For all patients with bothersome chronic tinnitus and high scores on the THI functional scale, fractal tones should be considered a promising initial sound therapy strategy. The findings from this open-label pilot study are preliminary, and further trials are needed before these results can be generalized to a larger tinnitus population.
\end{abstract}

\section{Introduction}

Tinnitus is a common, persistent symptom characterized by a sound sensation in the "ears" or "head" that is not produced by mechanical-acoustic signals from the external environment. ${ }^{1} \mathrm{~A}$ recent epidemiological study based on 1,960 interviews conducted in the city of São Paulo, Brazil, showed that $22 \%$ of the inhabitants had tinnitus, ${ }^{2}$ a higher prevalence than the $10-17 \%$ value commonly reported in the literature. ${ }^{3,4}$ We also observed

received

June 13, 2017

accepted

February 19, 2018

published online

March 29, 2018
DOI https://doi.org/

10.1055/s-0038-1635575. ISSN $1809-9777$. that the prevalence of the symptoms increases progressively with age and can be three times higher in the elderly (36\% over 65 years of age) than that in young adults (12\%). ${ }^{2}$ Many of the tinnitus sufferers also exhibited hearing loss and hypersensitivity to sound. ${ }^{5,6}$ Various pathophysiological causes underlie tinnitus, and all of the associated symptoms interfere with evaluations of affected patients. For most patients, medical management and appropriate counselling suffice; however, for other patients, the symptoms are persistent and bothersome,
Copyright (e 2018 by Thieme Revinter

Publicações Ltda, Rio de Janeiro, Brazil
License terms

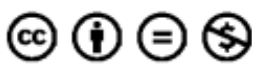


and they interfere with daily activities and with the quality of life. A recent U.S. study found that nearly a quarter of those who reported tinnitus symptoms described it as disabling, ${ }^{7}$ and according to Brazilian data, 34\% of cases are severely annoying. 2

The habituation theory ${ }^{8}$ and the neurophysiological model of tinnitus proposed by Jastreboff in 1990 provided new approaches for the clinical treatment of patients with tinnitus. Several therapeutic approaches have been proposed based on these models, including TRT (tinnitus retraining therapy), TAT (tinnitus therapy activities) and many other protocols that combine counselling, sound therapy and relaxation. ${ }^{9,10}$ These approaches combine sound therapy (as a facilitator) with procedures to habituate the reactions caused by tinnitus and its perception, thereby resulting in a decreased conscious perception of the signal. Sound therapy, such as sound amplification or the use of sound generators, aims to decrease the contrast in quiet environments and promote sound enrichment to facilitate habituation and provide partial or total masking of tinnitus.

Currently, different forms of sound therapy are available, and several forms of stimuli can be delivered from sound devices, which can be combined with amplification. Tinnitus primarily occurs because of sensory deprivation and increased central neural activity. A hearing aid can restore this system and is an effective treatment that has been confirmed by several studies. Other studies have revealed that music has great therapeutic potential for tinnitus relief. ${ }^{11,12}$ Listening to music leads to relaxation and stress relief, both of which can contribute to tinnitus control. In patients suffering from tinnitus, the presence of an annoying, persistent, and uncontrolled out-of-context sound signal can increase stress levels and cause an emotional disturbance that can exacerbate the perception of tinnitus. Neuroimaging has revealed the brain structures involved in tinnitus perception, which include the hippocampus, limbic area, frontal lobe, and cerebellum. These areas are also highly activated when listening to music. ${ }^{13-15}$ Music can be customized ${ }^{16}$ and filtered in accordance with the individual's hearing loss and tinnitus pitch and listening to pre-recorded music provides the patients with a structured listening protocol.

Fractal tones are harmonic and melodic tones that sound like "wind chimes"; however, because they are not predictable, these tones cannot be associated with any other conventional music to which the patient may have been previously exposed. This unpredictability is a unique and desirable feature of fractal tone music sound therapy because it induces passive listening, which is required for habituation. Habituation represents a natural brain function designed to reduce neutral stimulus perception under repeated exposure. Recent studies have shown that the use of fractal sounds may represent a viable alternative for minimizing the annoyance caused by tinnitus. ${ }^{17}$ Fractal tones appear to lead the listener to a pleasant and relaxing auditory experience and induce greater relaxation and stress relief compared with conventional music. In an open-label clinical trial, 14 hearing-impaired adults with a primary complaint of tinnitus were provided with one of several programs, including amplification only, fractal tones only, and a combination of amplification, white noise and/or fractal tones. Although the results did not show that the use of fractal tones was advantageous over the other treatment protocols, the patients preferred listening to those tones rather than noise over long periods. However, the role of fractal tones alone on chronic tinnitus sufferers is difficult to determine based on the study reported above ${ }^{17}$ because the report did not include the number of patients that used the fractal device or amplification alone, and the results were too similar among the four studied groups.

Music-based sound therapies have recently become a focus of tinnitus treatment because they target two of the mechanisms that are assumed to underlie tinnitus: perception and reaction. Such targeting has not been previously attributed to a white noise device. The few publications that have studied the therapeutic use of fractal tones among chronic tinnitus patients have rarely investigated the contribution of fractals separately from other techniques, such as amplification, white noise, cognitive behavioral intervention, and relaxation exercises. ${ }^{17-21}$

\section{Objectives}

The aim of this study was to determine the benefits of fractal tones as a unique sound therapy for treating chronic tinnitus.

\section{Patients and Methods}

The study was conducted at the Tinnitus Research Group of the Otolaryngology Department at the Clinical Hospital of the School of Medicine of Universidade de São Paulo. The sample consisted of subjects with normal listening ability and tinnitus sufferers who had mild hearing loss but no hearing loss complaints. These subjects were not using any other treatment or medication during the investigation. The participants were selected from August of 2014 to December of 2015, and they signed an informed consent and agreed to participate in the study.

\section{Inclusion Criteria}

The inclusion criteria were as follows:

- Normal listeners

- Mild sensorineural hearing loss (no hearing difficulties) that did not exceed $40 \mathrm{dBHL}$ from 250 to $8,000 \mathrm{~Hz}$

- Chronic tinnitus that has persisted for more than 6 months

- Tinnitus annoyance and handicap discomfort that are moderate, severe, or catastrophic as measured by the Tinnitus Handicap Inventory (THI, which was translated into and validated using the Portuguese language ${ }^{22}$ )

\section{Exclusion Criteria}

The exclusion criteria were as follows:

- Pulsatile and/or somatosensory tinnitus

- Patients with psychiatric disorders

- Individuals with motor, visual and/or cognitive functions that prevent the implementation of the procedures and the handling of hearing aids

- Patients prescribed antidepressants, anxiety drugs or sleeping pills

- History of drug abuse 


\section{Methods}

This study was approved by the Ethics Committee of the School of Medicine - Universidade de São Paulo - protocol number 611.174.

The participants were subjected to anamnesis, ENT (ear nose and throat) physical examination, and a complete audiological evaluation, which included pure tone audiometry by air and bone conduction at frequencies from $250 \mathrm{~Hz}$ to $8,000 \mathrm{~Hz}$ as well as speech audiometry and acoustic immittance audiometry test measures. In addition to the audiological evaluation, the participants underwent pitch and loudness matching and minimum masking level (MML) measurements. These measurements were used to check for changes in the perception of tinnitus before and after fitting the device. Many other studies, including previous fractal studies, have not used psychoacoustic measures of tinnitus. ${ }^{20,21}$ Our research team protocol uses these psychoacoustic measures routinely for all patients on their first visit and after treatments, especially sound therapy. The tinnitus impact and hearing handicap were assessed using the THI, HHIA (Hearing Handicap Inventory for Adults) ${ }^{23}$ and VAS (visual analog scale).

All subjects were administered instructional counselling at the beginning of the study. Comprehensive information on the basic hearing anatomy and physiology, the tinnitus etiology (that is, a natural consequence of hearing loss), the usual tinnitus course, the strong relationships between hearing and emotions, the potential negative associations with regard to hearing loss and the habituation process were also explained.

The fractal tones were delivered using MENU behind-theear hearing aids (Widex, Lynge, Denmark). All participants were fitted with the devices in both ears, and all wore them as open fittings, meaning that their ears were as open as possible to minimize the effects of occlusion and physical discomfort.

Of the five available styles of fractal tones (one of the four Widex Zen program components), the "Aqua style" was chosen because it was preferred by every participant during the fitting session. Amplification was not provided. The participants were instructed to use the device as frequently as possible and for at least 8 hours each day depending on their level of comfort with the device. The participants were also instructed to use the device during sleep. Adjustments to the fractal tones were performed by the audiologist to ensure that the tones were audible in quiet environments and did not interfere with conversational speech. To ensure that the device was used effectively and guide the participants regarding the device's ability to control tinnitus perception, we followed the participants for at least 6 months via individual counselling sessions.

The participants answered the questionnaires and were scored on the VAS scale at baseline, 1 month, 3 months and 6 months. The participants' hearing aid data log was checked at the meetings to certify that the device was continually used. Tinnitus pitch and loudness matching was performed before fitting and after 6 months of use.

The study involved ${ }^{1}$ an audiologist, who was responsible for fitting the devices; ${ }^{2}$ a physician, who was responsible for administering the clinical evaluations and questionnaires;
Table 1 Etiology of subjects

\begin{tabular}{|l|l|}
\hline Subjects & Etiology \\
\hline 1 & Post-traumatic stress disorder \\
\hline 2 & Metabolic syndrome \\
\hline 3 & Endolymphatic hydrops \\
\hline 4 & Otitis media \\
\hline 5 & Idiopathic \\
\hline 6 & Acoustic Trauma \\
\hline
\end{tabular}

and $^{3}$ a second audiologist, who conducted all audiological tests and psychoacoustic measures of tinnitus.

The variables sex, age, and time of tinnitus were described according to their frequency. The quantitative variables (THI, VAS, pitch/loudness, and LMM) were described as means and standard deviations (SDs). The statistical associations for each situation (before and after device use) were compared using the Student $t$-test for quantitative variables, and the statistical significance was set at $p<0.05$.

\section{Results}

Twelve participants were recruited, although six could not be assigned because they were already taking medicine for tinnitus treatment or had another acoustic stimuli preference. Four females and two males (mean age, 52 years; range, 34 to 70 years) were enrolled, and five participants completed the data collection. - Table 1 shows the etiology in each case. Two participants complained of tinnitus in one ear only, and the other four complained of a tinnitus sensation in both ears or in their heads. All subjects had tinnitus for more than 1 year, and three had tinnitus for more than 5 years. Although hearing loss had occurred in several subjects, it was not a complaint and did not exceed $40 \mathrm{dBHL}$ at any frequency. This finding was confirmed by the HHIA scale instrument, which was used to determine hearing loss that may represent a contributing factor to tinnitus annoyance. The HHIA score among almost all participants was less than 12, indicating no hearing difficulties. In - Fig. 1, audiograms of our sample are presented.

The tinnitus pitch, loudness, and MMLs were measured at baseline. The mean pitch was $6,033 \mathrm{~Hz}$ (range, 500 to $11,200 \mathrm{~Hz} ; \mathrm{SD}, 4,462 \mathrm{~Hz}$ ). The mean loudness was $9.8 \mathrm{dBSL}$ (range, 5 to $21 \mathrm{dBSL}$; SD, $6.2 \mathrm{dBSL}$ ). The mean MML was $8 \mathrm{dBSL}$ (range, 2 to $20 \mathrm{dBSL}$; SD, $6.3 \mathrm{dBSL}$ ). After 6 months of using the fractal tone device, all measurements were performed again. Results showed a mean pitch equal to $5,240 \mathrm{~Hz}$ (range, 500 to $11,200 \mathrm{~Hz}$; SD, 4,708 Hz), a mean loudness equal to $7 \mathrm{dBSL}$ (range, 5 to $12 \mathrm{dBSL}$; SD, $2.9 \mathrm{dBSL}$ ), and a mean MML of $7 \mathrm{dBSL}$ (range, 3 to $15 \mathrm{dBSL}$; SD, $4.7 \mathrm{dBSL}$ ). None of the following measurements showed significant improvements at the end of the treatment: pitch (paired $t$-test; two-tailed $p$ value $=0.3739$ ), loudness (paired $t$-test; two-tailed $p$ value $=0.3796$ ), and MML (paired $t$-test; two-tailed $p$ value $=0.1302$ ). The mean values, SDs and results are illustrated in - Table 2. 

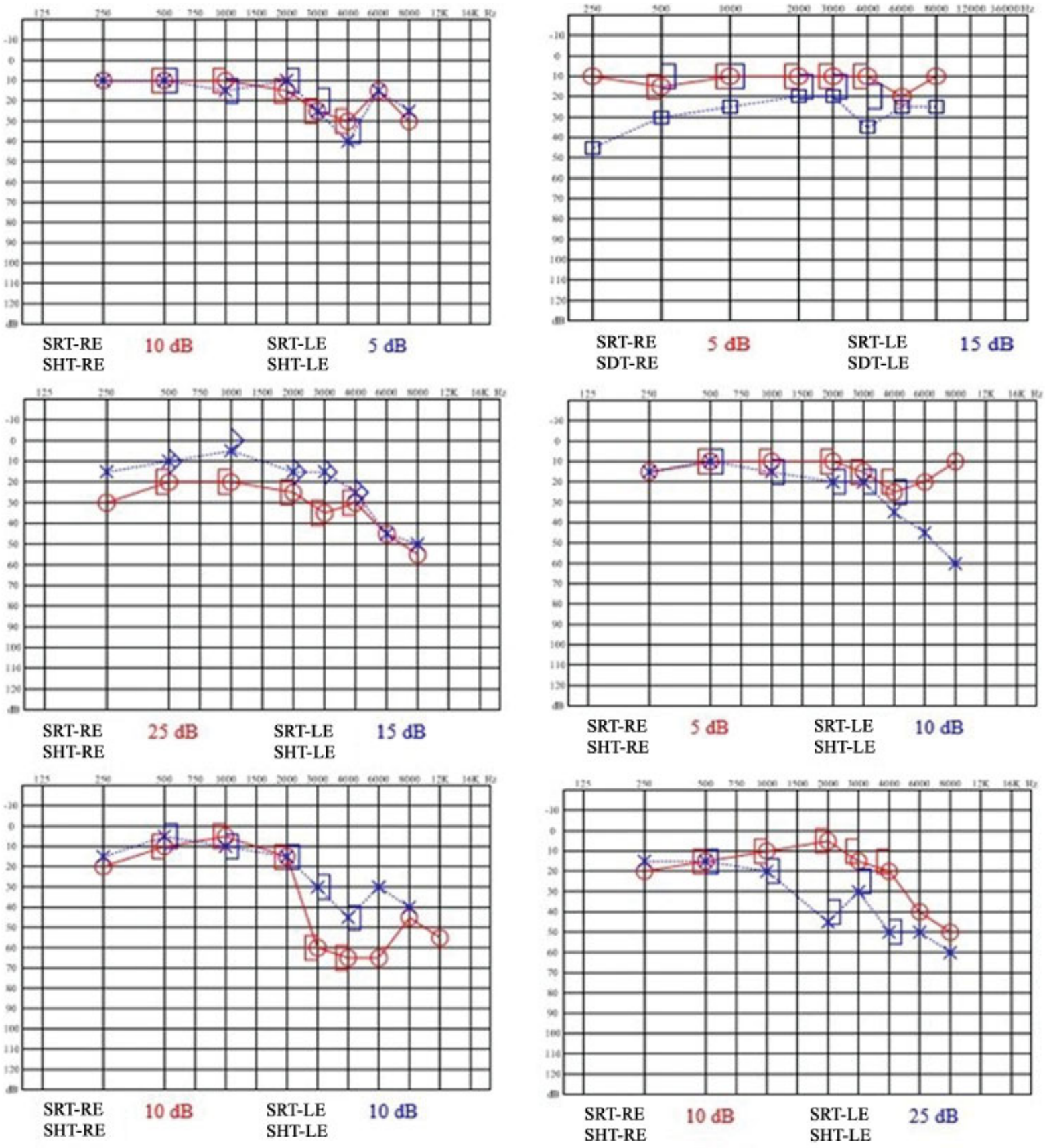

Fig. 1 Audiograms of the six participants in the fractal study. Abbreviations: dB, decibels; LE, left ear; RE, right ear; SDT, speech detective threshold; SHT, speech hearing threshold; SRT, speech reception threshold.

Table 2 Mean, standard deviation and loudness, pitch and minimum masking level values obtained at baseline and after 6 months

\begin{tabular}{|l|l|l|l|l|l|l|l|l|}
\hline & \multicolumn{2}{l}{ VAS } & \multicolumn{2}{l|}{ Loudness* $^{*}$} & \multicolumn{2}{l|}{ Pitch $^{\text {** }}$} & MML $^{*}$ \\
\cline { 2 - 10 } & A0 & A6 & A0 & A6 & A0 & A6 & A0 & A6 \\
\hline Mean & 7 & 5.6 & 9.8 & 7 & 6033 & 5240 & 8 & 7 \\
\hline SD & 0.8 & 1.3 & 6.2 & 2.9 & 4462 & 4708 & 6.3 & 4.7 \\
\hline$P$ & & 0.1087 & & 0.3796 & & 0.3739 & & 0.1302 \\
\hline MSD & & No & & No & & No & & No \\
\hline
\end{tabular}

Abbreviations: A0, at baseline; A6, after 6 months of device use; MSD, mean significant difference at $p<0.05$; , $t$-test $\mathrm{p}$ value; SD, standard deviation; VAS, visual analog scale.

* = measured in $\mathrm{dBSL}$ (decibels, that is, sensation level)

$* *$ measured in $\mathrm{Hz}$. 
Fractal Tones Effect for Tinnitus Simonetti et al. 391

Table 3 Mean, standard deviation and differences for the THI and its subscales at baseline and after 6 months

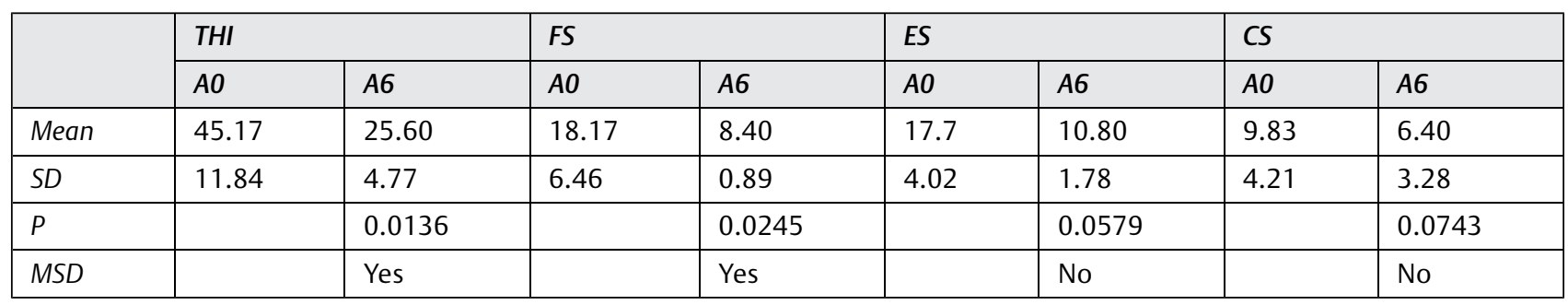

Abbreviations: A0, at baseline; A6, after 6 months of device use; CS, catastrophic score; ES, emotional score; FS, functional score; MSD, mean significant difference at $p<0.05 ; \mathrm{P}$, $t$-test $\mathrm{p}$ value; SD, standard deviation; THI, tinnitus handicap inventory.

At baseline, the mean VAS score was 7 (range, 6 to 8), and after 6 months of treatment, the mean VAS score was 5.6 (range, 4 to 7 ). Although a slight improvement was observed for all subjects, the results were not statistically different (paired $t$-test, two-tailed $p$ value $=0.1087$ ).

The mean THI measured at baseline was 45 (range, 30 to 66), and the final THI was 25 (range, 18 to 30 ). A paired sample $t$-test showed that the 20-point difference was statistically significant $(p<0.05)$. The SDs, significant differences and confidence intervals are shown in - Table 3. -Fig. 2 illustrates the measured values.

The following three THI subscales were also analyzed: functional, emotional, and catastrophic factors. All of these aspects improved after 6 months of treatment; however, only the functional aspect showed a statistically significant difference. The results are also shown in -Table 3 and -Figs. 2-3.

\section{Discussion}

A self-reported survey using the 1999-2004 National Health and Nutrition Examination Surveys (NHANES) database showed that more than 14,000 subjects among the U.S. adult population reported having tinnitus. Among nine assessed tinnitus treatment methods (hearing aids, music, medication, relaxation exercises, counselling, non-wearable sound gen-

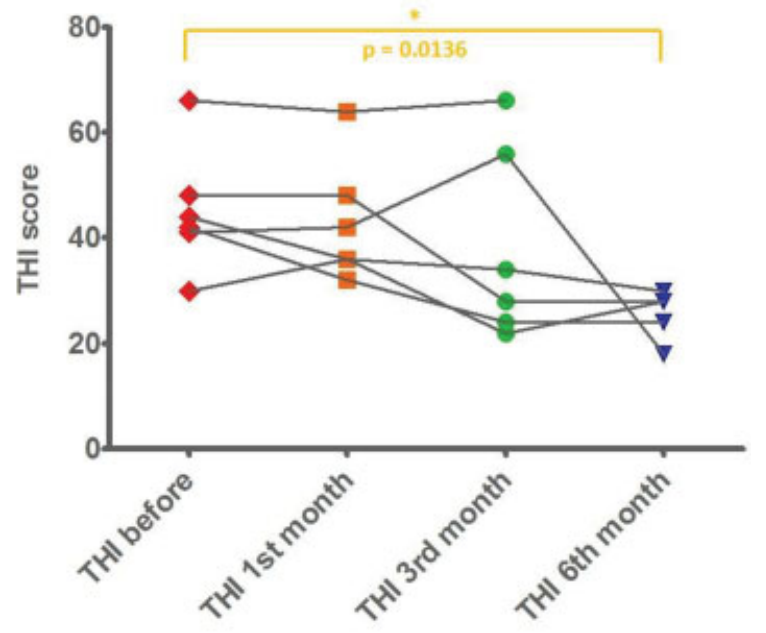

Fig. 2 Tinnitus handicap inventory scores measured before and after 1,3 and 6 months of fractal therapy. ${ }^{*}$, significant difference $(p<0.05)$. erators, herbs and dietary supplements, wearable sound generators, and psychological counselling), substantial tinnitus amelioration was only observed for the treatments with hearing aids (34\%) and music (30\%). ${ }^{7}$ This finding was one of the motivations for our investigation into the effects of music on chronic tinnitus sufferers.

The first report of the use of music for tinnitus management was published in 1988; in this study, music and different types of tinnitus maskers were compared, and music was recommended over traditional maskers for the first time. ${ }^{24}$ However, music did not achieve widespread use as a form of sound therapy until recently. ${ }^{16,25,26}$ Neuromonics is one of the first tinnitus music sound therapies to use pre-recorded customized music (increased intensity) in accordance with the individual's hearing loss and tinnitus pitch. ${ }^{16}$ Another sound-based music technique is the tailor-made notched music training endeavor, which presents an opposite approach to that of Neuromonics because it removes the tinnitus frequencies and neighboring frequency regions from the music. ${ }^{26}$ Fractal tones have a sound that is similar to wind chimes, and which can be processed using an algorithm to create a melodic chain of tones that are sufficiently repetitive to sound familiar and sufficiently varied to avoid predictability. ${ }^{27}$ In addition, fractal tones emphasize certain musical elements, such as a slow onset, slower tempo (near the resting heart rate of $\sim 60-72$ beats/minute), lower pitch, repetition, non-emotional content (to avoid evoking memories), and the combination of these elements produce a soothing effect and heightened concentration in certain individuals. ${ }^{28}$

Thus, ENT doctors and audiologists can select from a wide variety of tinnitus music sound therapy styles (for example, fractal tones, Neuromonics, tailor-made notched music), although the styles that best suit each type of tinnitus patient are unknown. Therefore, our aim was to verify the effectiveness of fractal tones on tinnitus perception, annoyance, and magnitude among chronic tinnitus sufferers recruited from the Tinnitus Research Group of the Otolaryngology Department of the Medical School at Universidade de São Paulo. Patients with moderate, severe, or catastrophic chronic tinnitus, as measured by THI, were selected. These patients had experienced a positive impact on their quality of life but had not complained of significant hearing loss as measured by the HHIA. Moreover, these patients were likely to benefit from sound therapy without amplification because our goal was to verify the effect of fractal tones alone. 

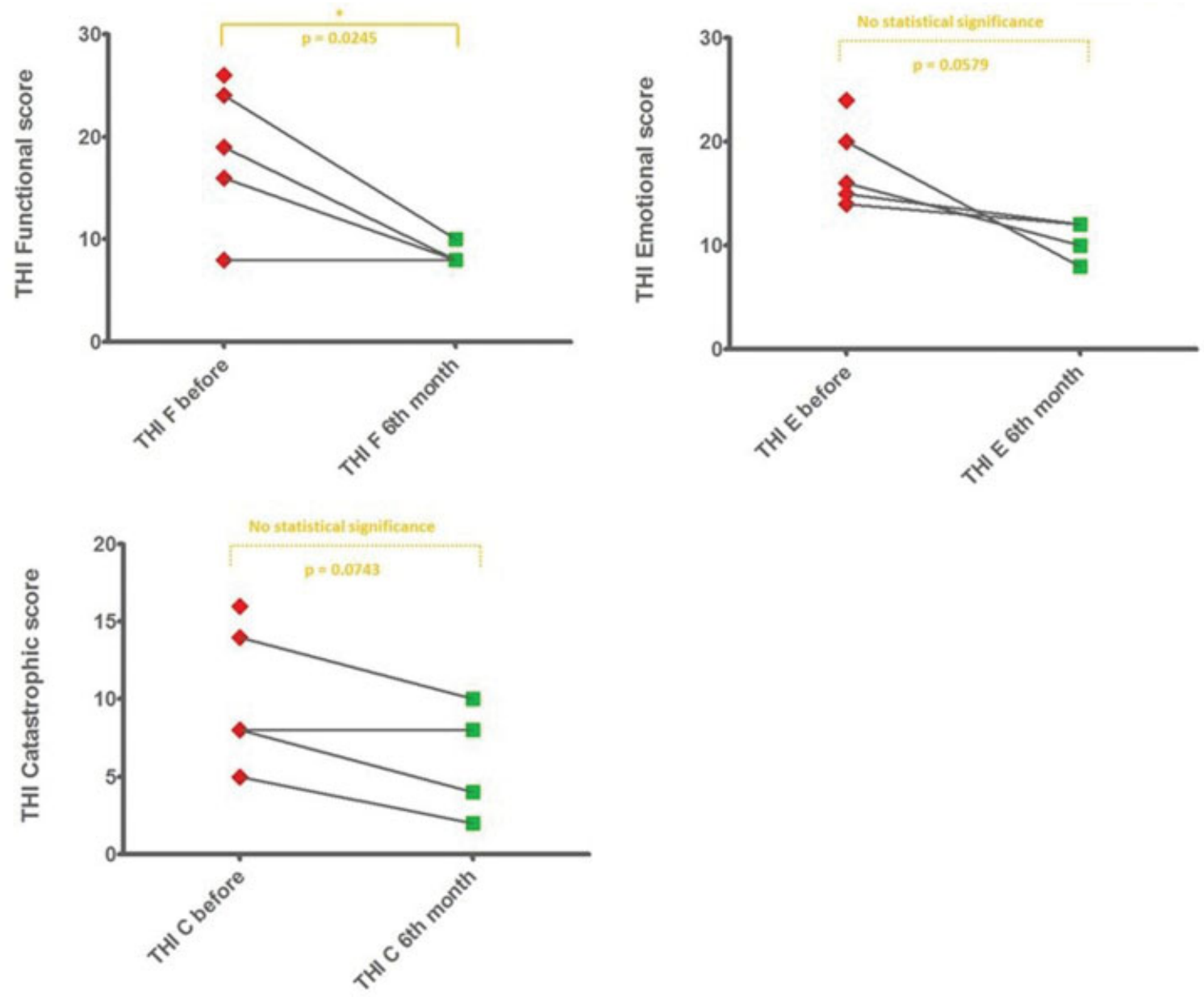

Fig. 3 Tinnitus handicap inventory functional, emotional and catastrophic scores measured at baseline and after 6 months of fractal therapy. ${ }^{*}$, significant difference $(p<0.05)$.

Among the excluded tinnitus patients, one was a musician who dropped out because due to considering fractal tones to be annoying. This reasoning was consistent with the results of a previous similar report ${ }^{17}$ because a universal sound therapy that suits and works for all tinnitus patients has not been identified, and certain types of music stimuli induce relaxation in some individuals but not others. ${ }^{28}$

We followed most of the assigned patients for 6 months and others for 1 year. All patients were instructed to listen to the fractal tones during waking hours, and our data log analysis showed that the fractal tones were used for at least 8 hours per day on average. The recommended time frame of 8 hours per day is inconsistent with the recommendations in other studies, in which the recommended use of fractal tones was only for 2 or 4 hours each day. ${ }^{17}$ After a 6 -month period, certain patients decided to keep the device because they were using it throughout the day. However, other patients who did not observe considerable improvements or tinnitus suppression stopped using the device continuously after the end of the study. After the 6-month period, certain patients had worsened hearing thresholds and were subsequently fitted with a hearing aid amplification, which represented the best initial time point for selecting the combined hearing aid/sound therapy device. During the study period, only the
Aqua fractal style was used per the participants' choice. This style was also the most preferred by participants in another study. ${ }^{18}$

After the sound therapy period, certain patients indicated experiencing a clinical improvement in their daily quality of life, which included better concentration while reading or studying and a greater ability to relax. Two participants used the device to sleep because sleep problems were among their main complaints. All six participants (100\%) showed a clinical significant improvement in their THI scores, which decreased by almost 20 points (the mean score at baseline was 45.1 and the mean score after 6 months of sound therapy was 25.6), and this finding is consistent with a previous report in which a 30-point improvement in THI was observed. ${ }^{20}$ Previous studies have indicated that 20-point THI score changes are clinically significant. ${ }^{29}$ According to our data, the THI changes were only clinically significant at the final appointment after 6 months of therapy. These findings are inconsistent with those of previous studies in which an earlier response was observed at 2 months. ${ }^{20,21}$ The authors applied the Zen therapy, which includes the four components: fractal tones, broadband amplification, relaxation exercises and sleep management, in 24 and 19 assigned participants subjected to THI and TFI (tinnitus functional 
index) measurements, respectively. In that study, the post hoc analysis based on a paired sample $t$-test showed a significant score reduction at 2 months, which indicated that earlier tinnitus distress resolution and habituation could be achieved by the Zen therapy. However, the authors used a simultaneous combination of tools, and the contributions of each tool to the observed tinnitus improvement is unclear. Based on our results, we aimed to determine the specific benefits of fractal tones for tinnitus sufferers without combining it with other therapeutic strategies, and our instructions recommended the use of this therapy for at least 8 hours each day.

In another study that was similar to ours, ${ }^{30}$ the author investigated the effectiveness of fractal tones in patients with minimal hearing loss by comparing two groups $(n=19 / 22)$ with the same tinnitus characteristics (THI $>18$ ), and the control group only received counselling. For 2 months, only fractal tones were employed in the study group, although amplification was subsequently provided for those who had not been responsive to fractal tones, and who indicated that they experienced hearing loss. For those with normal hearing, other components of Widex Zen Therapy (WZT) were added, such as cognitive behavioral therapy and relaxation exercises. Clinical and statistically significant improvements were observed in the study group (21 points on the TFI) for the first 2 months, and the patients continued to improve until the 6th month. The author also investigated the longterm effects at 6 months after the end of the treatment and verified that the participants who continued to use the device maintained the benefits of treatment, whereas those who stopped using the device did not maintain any benefits. This finding was also observed in several of our participants.

The THI is a 25-item self-reporting instrument that is grouped into three subscales or domains. The functional subscale reflects limitations experienced by the patient in mental, social/occupational, and physical functioning areas; the emotional subscale includes a broad range of emotional reactions to tinnitus; and the catastrophic subscale includes the destructive effects of experiencing tinnitus. We determined the subscales or domains that were responsible for the THI score improvement after the 6-month fractal sound therapy, and our results indicated that the primary result of this type of sound therapy was a decrease in the scores on the THI functional scale, which measures concentration, reading, attention, consciousness, sleep, social activities, and household tasks. This finding was surprising and inconsistent with previous studies in which fractal tones were reported to promote improvements in the emotional and catastrophic responses. ${ }^{19-21}$ In our study, no statistically significant changes were observed in the results for the THI emotional and catastrophic domains recorded at baseline and at the 6-month evaluation.

The VAS, pitch and loudness matching, and MMLs showed a slight tendency toward improvement in certain participants; however, our limited sample size precluded the detection of clinically significant differences in these measures or any type of improvement in the tinnitus magnitude after 6 months of fractal sound therapy. Because tinnitus can exacerbate stress (and vice versa), relaxation and proper sleep schedules are important for tinnitus patients, who are instructed to continue with their lives and common routine. Although we did not expect to observe drastic changes in the psychoacoustic measures, we did expect to observe changes in the participants' reactions to tinnitus and the impact of tinnitus on their quality of life. These changes were detected by the THI, which indicated that the greatest changes occurred in the functional domain, as well as by the participants' spontaneous reports regarding improvements in their attention and concentration.

Many different stimuli can be delivered by different devices used in sound therapy. Such therapies can be applied in various strategies to control tinnitus. For example, these therapies can be used to mask the tinnitus, avoid silence, diminish contrast, thus facilitating tinnitus management. However, these therapies are not effective for every patient, and the research remains inconclusive.

Tinnitus has different underlying causes; therefore, every patient and treatment strategy are unique. Fractal tones are recommended as a sound therapy because this technique clearly helped participants to concentrate and manage the associated distress, and it facilitated sleep in certain participants.

We suggest that a combination of fractal tones with other elements, such as relaxation techniques, will be beneficial for tinnitus sufferers.

The results of our study support the use of fractal tones as a sound therapy for tinnitus patients. We also recommend future research on the long-term effects of this therapy.

\section{Conclusions}

We traced the individual contributions of fractal tones on chronic tinnitus sufferers and found that the main benefit of this approach was in the THI functional domain, which includes concentration, reading, attention, consciousness, sleep, social activities, and household tasks. For all patients with bothersome chronic tinnitus who have high scores on the THI functional scale, fractal tones should be considered as a good initial sound therapy. The findings of this open-label pilot study are preliminary, and other trials must be conducted before these results can be generalized to a larger tinnitus population.

\section{Conflict of Interest}

The authors declare that the research was conducted in the absence of any commercial or financial relationships that could be construed as a potential conflict of interest. The MENU hearing aids used as the fractal tone devices were donated by Widex Hearing aids.

\section{References}

1 Weisz N, Hartmann T, Dohrmann K, Schlee W, Norena A. Highfrequency tinnitus without hearing loss does not mean absence of deafferentation. Hear Res 2006;222(1-2):108-114

2 Oiticica J, Bittar RS. Tinnitus prevalence in the city of São Paulo. Rev Bras Otorrinolaringol (Engl Ed) 2015;81(02):167-176

3 Davis A, Refaie AE. Epidemiology of tinnitus. In: Tyler R, editor. Tinnitus Handbook. San Diego (CA): Singular Publishing Group; 2000:1-23 
4 Henry JA, Dennis KC, Schechter MA. General review of tinnitus: prevalence, mechanisms, effects, and management. J Speech Lang Hear Res 2005;48(05):1204-1235

5 Jastreboff P, Hazell JWP. Tinnitus Retraining Therapy: Implementing the Neurophysiological Model. Cambridge: Cambridge University Press; 2004:83-84

6 Tyler RS. Neurophysiological models, psychological models, and treatments for tinnitus. In: Tyler RS, editor. Tinnitus Treatments: Clinical Protocols. New York: Thieme Medical Publishers; 2006:2-23

7 Kochkin S, Tyler R, Born J. MarkeTrakVIII: Prevalence of tinnitus and efficacy of treatments. Hearing Rev 2011;18(12):10-26

8 Hallam RS. Tinnitus: Living with the ringing in your ears. New York: Harper Collins; 1989

9 Jastreboff PJ. Phantom auditory perception (tinnitus): mechanisms of generation and perception. Neurosci Res 1990;8(04):221-254

10 Jastreboff PW. Categories of the patients in TRT and the treatment outcome. In: Hazell JWP, editor. Proceedings of the Sixth International Tinnitus Seminar 1999. London: The Tinnitus and Hyperacusis Center; 1999:394-398

11 Attanasio G, Cartocci G, Covelli E, et al. The Mozart effect in patients suffering from tinnitus. Acta Otolaryngol 2012;132(11): 1172-1177

12 Hann D, Searchfield GD, Sanders M, Wise K. Strategies for the selection of music in the short-term management of mild tinnitus. Aust N Z J Audiol 2008;30(02):129-140

13 Lockwood AH, Salvi RJ, Coad ML, Towsley ML, Wack DS, Murphy BW. The functional neuroanatomy of tinnitus: evidence for limbic system links and neural plasticity. Neurology 1998;50 (01):114-120

14 Leaver AM, Seydell-Greenwald A, Turesky TK, Morgan S, Kim HJ, Rauschecker JP. Cortico-limbic morphology separates tinnitus from tinnitus distress. Front Syst Neurosci 2012;6:21

15 Simonetti P, Oiticica J. Tinnitus neural mechanisms and structural changes in the brain: the contribution of neuroimaging research. Int Arch Otorhinolaryngol 2015;19(03):259-265

16 Davis PB, Wilde RA, Steed LG, Hanley PJ. Treatment of tinnitus with a customized acoustic neural stimulus: a controlled clinical study. Ear Nose Throat J 2008;87(06):330-339
17 Sweetow RW, Sabes JH. Effects of acoustical stimuli delivered through hearing aids on tinnitus. J Am Acad Audiol 2010;21(07): 461-473

18 Kuk F, Peeters H, Lau CL. The efficacy of fractal music employed in hearing aids for tinnitus management. Hearing Rev 2010;17(10): $32-42$

19 Sweetow RW. The use of fractal tones in tinnitus patient management. Noise Health 2013;15(63):96-100

20 Herzfeld M, Ciurlia-Guy E, Sweetow R. Clinical trial on the effectiveness of Widex Zen Therapy for tinnitus the hearing. [Review]. 2014. Available at: http://www.hearingreview.com/ 2014/10/clinical-trial-effectiveness-widex-zen-therapy-tinnitus

21 Sweetow RW, Fehl M, Ramos PM. Do tinnitus patients continue to use amplification and sound therapy post habilitation? Hearing Rev 2015;21(03):34

22 Paula Erika Alves F, Cunha F, Onishi ET, Branco-Barreiro FCA Ganança FF. [Tinnitus Handicap Inventory: cross-cultural adaptation to Brazilian Portuguese]. Pro Fono 2005;17(03):303-310

23 Newman CW, Sandridge SA, Jacobson GP. Psychometric adequacy of the Tinnitus Handicap Inventory (THI) for evaluating treatment outcome. J Am Acad Audiol 1998;9(02):153-160

24 Al-Jassim AH. The use of Walkman Mini-stereo system as a tinnitus masker. J Laryngol Otol 1988;102(01):27-28

25 Davis PB, Paki B, Hanley PJ. Neuromonics Tinnitus Treatment: third clinical trial. Ear Hear 2007;28(02):242-259

26 Okamoto H, Stracke H, Stoll W, Pantev C. Listening to tailor-made notched music reduces tinnitus loudness and tinnitus-related auditory cortex activity. Proc Natl Acad Sci US A 2010;107(03):1207-1210

27 Hsü KJ, Hsü AJ. Fractal geometry of music. Proc Natl Acad Sci U S A 1990;87(03):938-941

28 Furnham A, Allass K. The influence of musical distraction of varying complexity on the cognitive performance of extroverts and introverts. Eur J Pers 1999;13(01):27-38

29 Newman CW, Weinstein BE, Jacobson GP, Hug GA. The Hearing Handicap Inventory for Adults: psychometric adequacy and audiometric correlates. Ear Hear 1990;11(06):430-433

30 Sweetow R, Kuk F, Caporali S. A controlled study on the effectiveness of fractal tones on subjects with minimal need for amplification. Hearing Rev 2015;22(09):30 